\title{
Brain Perfusion in Encephalopathic Newborns after Therapeutic Hypothermia
}

\author{
A. N. Massaro, M. Bouyssi-Kobar, T. Chang, L. G. Vezina, A. J. du Plessis, and C. Limperopoulos
}

\begin{abstract}
BACKGROUND AND PURPOSE: Cerebral perfusion patterns in neonates with HIE after therapeutic hypothermia have not been well described. The objectives of this study were to compare global and regional perfusion between infants with HIE and neonate controls and to relate measures of cerebral perfusion to brain injury on conventional MR imaging in neonates with HIE.
\end{abstract}

MATERIALS AND METHODS: Term encephalopathic neonates meeting criteria for hypothermia between June 2011 and January 2012 were enrolled in this prospective observational study. MR imaging-ASL was performed in the second week of life. Comparisons were made with data from neonate controls who underwent the same imaging protocol. NIRS measures of cerebral oxygenation during and immediately after hypothermia were also evaluated in a subset of patients. Secondary analyses were performed to assess cerebral perfusion and oxygenation differences by pattern of injury on qualitative MR imaging interpretation.

RESULTS: We enrolled 18 infants with HIE and 18 control infants. Mean global CBF and regional CBF in the basal ganglia, thalamus, and anterior white matter were higher in cases compared with controls. Infants with HIE with injury on MR imaging, however, had lower CBF (significant in the thalamus) compared with those with normal MR imaging. Decreased FTOE by NIRS further differentiated patients with HIE with injury on MR imaging.

CONCLUSIONS: Disturbed cerebral perfusion is observed in the second week of life in some babies with HIE despite treatment with hypothermia. Infants with HIE with injury on MR imaging have lower regional CBF in the thalamus compared with those without injury, possibly representing pseudonormalization of $\mathrm{CBF}$ and low metabolic demand after progression to irreversible brain injury.

ABBREVIATIONS: $\mathrm{HIE}=$ hypoxic-ischemic encephalopathy; $\mathrm{EEG}$ = electroencephalogram; NIRS = near-infrared spectroscopy; FTOE = fractional tissue oxygen extraction; $\mathrm{SaO}_{2}=$ arterial oxygen saturation

$\mathrm{N}$ eonatal HIE is a devastating cause of mortality and neurologic disability despite incorporation of therapeutic hypothermia into standard care. ${ }^{1-4}$ Efforts to further improve adverse outcomes after treatment with hypothermia are needed. The du-

Received August 10, 2012; accepted after revision October 6.

From the Departments of Neonatology (A.N.M.), Fetal and Transitional Medicine (A.N.M., A.J.d.P., C.L.), Diagnostic Imaging and Radiology (L.G.V., C.L.), and Neurology (T.C., A.J.d.P.), and Advanced Pediatric Brain Imaging Research Laboratory (M.B.-K., C.L.), Children's National Medical Center, Washington DC.

This project was supported by Award Nos. ULIRR031988 and KL2 RR031987 from the National Institutes of Health National Center for Research Resources. Its contents are solely the responsibility of the authors and do not necessarily represent the official views of the National Center for Research Resources or the National Institutes of Health This work was also supported by the Canadian Institutes of Healthy Research (MOP-81116, to C.L.).

Please address correspondence to An N. Massaro, MD, The George Washington University School of Medicine, Department of Neonatology, Children's National Medical Center, 111 Michigan Ave, NW, Washington, DC 20010; e-mail: anguyenm@cnmc.org

-- Indicates open access to non-subscribers at www.ajnr.org

http://dx.doi.org/10.3174/ajnr.A3422 ration of the true therapeutic window in affected neonates is not known. ${ }^{5}$ Advances toward adjuvant neurotherapeutics have been limited by this incomplete knowledge about the presence and timing of secondary injurious processes (eg, excitotoxicity, proinflammatory cascades, oxidative injury) occurring during and after reperfusion. ${ }^{6}$ Evaluation of cerebral blood flow after treatment with hypothermia may provide insight into continuing physiologic alterations that may signify ongoing injury in select neonates.

MR imaging-ASL provides a noninvasive quantitative assessment of cerebral perfusion. ${ }^{7}$ It offers advantages over other methods to interrogate cerebral blood flow such as cerebral artery Doppler or NIRS that lack anatomic resolution and other imaging modalities such as positron-emission tomography or xenon-enhanced CT, which require injection of contrast material and exposure to ionizing radiation. ${ }^{8}$

Prior studies with the use of alternative methods to interrogate cerebral perfusion have demonstrated an initial decrease in CBF after asphyxia, ${ }^{9,10}$ followed by a relative hyperemia during the 
first 1 to 5 days of life. ${ }^{11-16}$ The pattern and duration of this hyperemia remain poorly understood because most studies to date have been confined to the first week after injury. Furthermore, the relationship between perfusion disturbances and injury by conventional MR imaging has not been previously investigated. To gain better insight into the longer-term cerebral perfusion patterns after hypoxia-ischemia, the current study investigated patterns of CBF in hypothermia-treated neonates with HIE in the second week of life. Through the use of MR imaging-ASL, we aimed to 1) compare global and regional perfusion between infants with HIE and healthy neonate controls and 2) relate measures of cerebral perfusion to brain injury on conventional MR imaging in neonates with HIE. As a secondary aim, we measured cerebral oxygenation by NIRS during and immediately after hypothermia in a subset of patients and related these early measures of cerebral oxygenation to later cerebral perfusion patterns.

\section{MATERIALS AND METHODS Study Population}

Term neonates with HIE transferred to a level IIIC neonatal intensive care unit for therapeutic hypothermia between June 2011 and January 2012 were enrolled in this prospective, observational study. Inclusion criteria included any encephalopathic neonate meeting established National Institute of Child Health and $\mathrm{Hu}-$ man Development Neonatal Research Network criteria (ie, infants were $>36$ weeks' gestational age, $>1800 \mathrm{~g}$ at birth, demonstrated metabolic acidosis and/or low Apgar scores, and exhibited signs of moderate to severe clinical encephalopathy). Infants were treated with whole-body hypothermia according to the established protocol. ${ }^{1}$ Infants who underwent MR imaging-ASL after hypothermia (added to our routine imaging protocol after June 2011) were included in this report. Exclusion criteria included major congenital anomalies, known or suspected chromosomal abnormalities, and major brain malformations or non-HIE injury on qualitative imaging. The study was approved by the institutional review board, and a written informed consent was obtained from the parent(s) of each participant before imaging. Comparisons with an anonymized dataset of healthy neonate controls were made. Control neonates were concurrently enrolled for a separate prospective study and imaged with the same protocol as used in the subjects, without use of sedation, after informed consent was obtained. All data were collected in compliance with Health Information Portability and Accountability Act regulations.

\section{Clinical Data Collection}

Clinical and demographic data were collected from the birth hospital records. Clinical encephalopathy grade was classified according to modified Sarnat criteria. ${ }^{1,17}$ Infants treated with hypothermia underwent routine continuous EEG monitoring with surface electrode placement according to the international 10-20 system modified for neonates. EEG monitoring was initiated as soon as possible after admission and continued through at least 12 hours after completion of rewarming. EEG background abnormality was assessed by a neonatal neurologist in the first 24 hours of life and again at rewarming and classified according to a previously described scoring system. ${ }^{18}$ The presence of electrographic seizures at any time during hypothermia was also noted. Covari- ables with potential to influence CBF at the time of MR imaging including use of sedation, hematocrit level, partial pressure of carbon dioxide, and patient temperature were also recorded.

\section{Near-Infrared Spectroscopy}

Cerebral tissue oxygen saturation by NIRS (FORE-SIGHT Cerebral Oximeter; Casmed, Branford, Connecticut) was monitored continuously during hypothermia treatment and up to 12 hours after rewarming. Cerebral tissue oxygen saturation and simultaneous transcutaneous $\mathrm{SaO}_{2}$ measurements were recorded hourly by bedside NICU nurses. These values were collected retrospectively from the clinical records for study patients. FTOE was calculated by use of the formula FTOE $=\left(\mathrm{SaO}_{2}-\right.$ cerebral tissue oxygen saturation) $/ \mathrm{SaO}_{2}$. Average FTOE values were calculated for two 12-hour periods of interest: during 12 to 24 hours of cooling (DAY 1) and during the 12-hour period after rewarming (DAY 4).

\section{Magnetic Resonance Imaging}

MR imaging was performed after rewarming in surviving infants according to clinical protocol during the second week of life (target age, 7-10 days) on a 3T scanner (Discovery MR750; GE Healthcare, Milwaukee, Wisconsin) with the use of a 32-channel receive-only head coil (MR Instruments, Minneapolis, Minnesota). Infants were imaged without sedation unless they were receiving sedative medications for clinical indications, were not enterally feeding to allow for the "feed and bundle" method for nonsedated neonatal MR imaging acquisition, or had failed a prior nonsedated MR imaging attempt. Standard anatomic sequences included 3D T1-weighted spoiled gradient-recalled (TE, $3.1 \mathrm{~ms}$; TR, $7.8 \mathrm{~ms}$; TI, $450 \mathrm{~ms}$; FOV, $220 \times 220 \mathrm{~mm}$; section thickness, $1 \mathrm{~mm}$ ), double acquisition axial FSE T2 proton attenuation (TE 1, 30 ms; TE 2, 150 ms; TR, 9000 ms; FOV, $192 \times 192$ $\mathrm{mm}$; section thickness, $3 \mathrm{~mm}$ ), axial T2 PROPELLER (TE, 83.72 ms; TR, 8677 ms; FOV, $256 \times 256 \mathrm{~mm}$; section thickness, $2 \mathrm{~mm}$ ), axial T2*-weighted susceptibility (TE, $25 \mathrm{~ms}$; TR, $46.9 \mathrm{~ms}$; FOV, $200 \times 200 \mathrm{~mm}$; section thickness, $2 \mathrm{~mm}$ ), coronal T1 FLAIR PROPELLER (TE, 38.6 ms; TR, 1525 ms; TI, $565.42 \mathrm{~ms}$; FOV, $256 \times 256 \mathrm{~mm}$; section thickness, $2 \mathrm{~mm}$ ), and axial 25-direction DTI (TE, $80 \mathrm{~ms}$; TR, $8000 \mathrm{~ms}$; FOV, $200 \times 200 \mathrm{~mm}$; section thickness, $3 \mathrm{~mm}$; b-value, 1000), with generation of ADC maps off-line.

An axial pseudocontinuous 3D ASL sequence was also performed according to routine protocol with the use of the following parameters: TE, $11.1 \mathrm{~ms}$; TR, $4326 \mathrm{~ms}$; FOV, $240 \times 240 \mathrm{~mm}$; section thickness, $3 \mathrm{~mm}$, spiral readout. CBF maps were generated with the use of Functool software (GE Healthcare) with the following quantification algorithm:

$$
\begin{aligned}
\mathrm{CBF}= & 6000 \lambda^{\star} \\
& \frac{\left(1-\exp \left(-\mathrm{ST}(\mathrm{s}) / \mathrm{T}_{1 \mathrm{t}}(\mathrm{s})\right)\right) \exp \left(\mathrm{PLD}(\mathrm{s}) / \mathrm{T}_{1 \mathrm{~b}}(\mathrm{~s})\right)}{2 \mathrm{~T}_{1 \mathrm{~b}}(\mathrm{~s})\left(1-\exp \left(-\mathrm{LT}(\mathrm{s}) / \mathrm{T}_{1 \mathrm{~b}}(\mathrm{~s})\right)\right) \varepsilon^{\star} \mathrm{NEX}_{\mathrm{pw}}} \\
& \star\left(\mathrm{PW} / \mathrm{SF}_{\mathrm{pw}} \mathrm{PD}\right)
\end{aligned}
$$

where partition coefficient $(\lambda)$ was set to whole-brain average of 0.9 , saturation time (ST) was set to 2 seconds, typical gray matter tissue $\mathrm{T} 1\left(\mathrm{~T}_{1 \mathrm{t}}\right)$ was 1.2 seconds, $\mathrm{T} 1$ of blood $\left(\mathrm{T}_{1 \mathrm{~b}}\right)$ was assumed to 

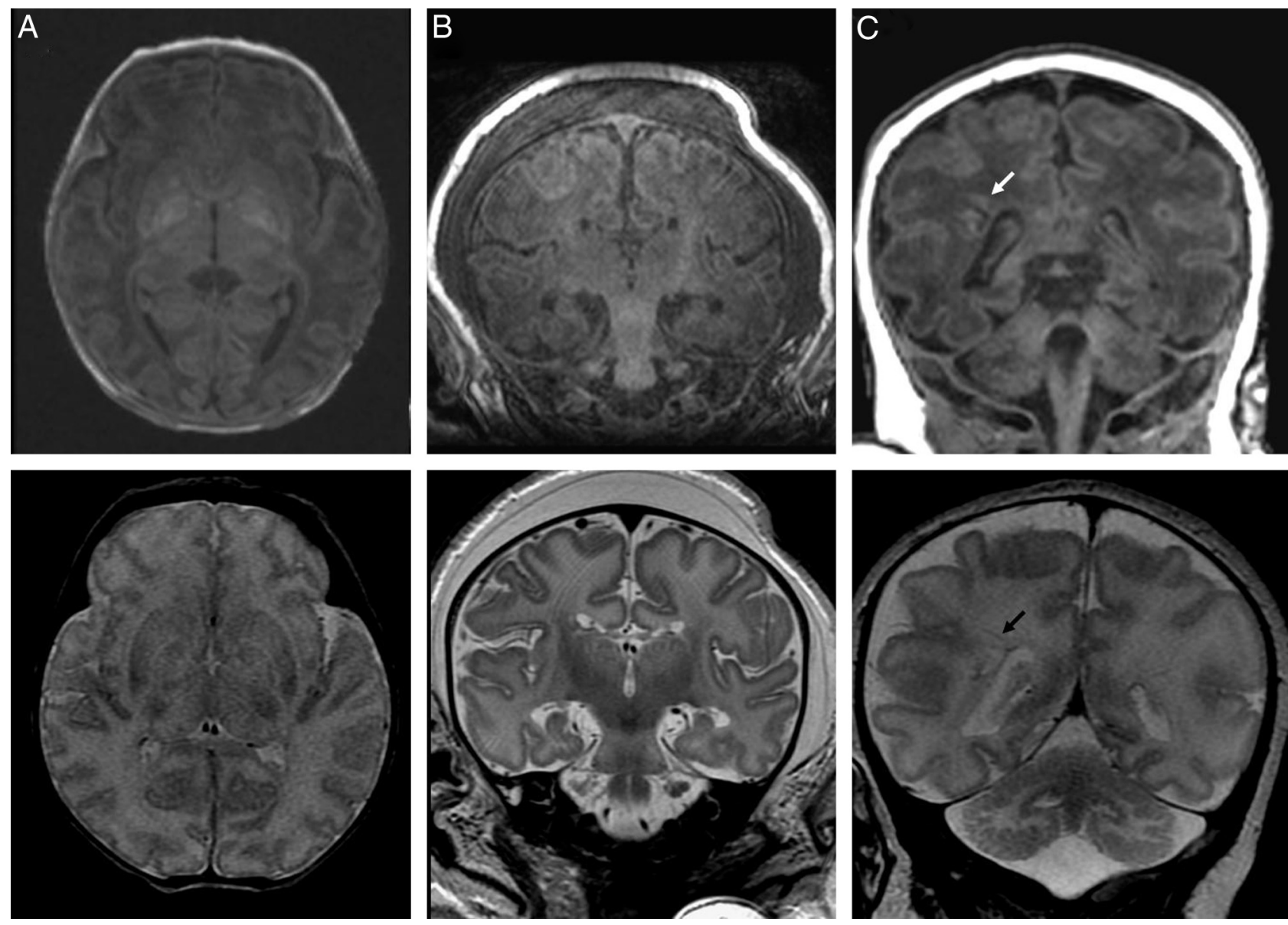

FIG 1. Pattern of injury scoring for infants with HIE. Representative examples of $A$, basal ganglia predominant; $B$, watershed; and $C$, focal/ multifocal injury (periatrial white matter injury at arrow) are shown.

be 1.6 seconds at $3 \mathrm{~T}$, postlabeling delay (PLD) was set to $1025 \mathrm{~ms}$, labeling duration (LT) was 1.5 seconds, overall efficiency $(\varepsilon)$ was $0.6, \mathrm{NEX}_{\mathrm{PW}}$ was 3 ; PW is the perfusion-weighted or raw difference image, $\mathrm{SF}_{\mathrm{pw}}$ is the scaling factor of the $\mathrm{PW}$ sequence, and $\mathrm{PD}$ was the partial saturation of the reference image. The labeling slab thickness was adjusted according to the size of the infant's head (range, 84-96 mm) and positioned at the base of the pons.

Qualitative images were reviewed by a neuroradiologist (G.V.) blinded to clinical data and were scored according to Barkovich et $\mathrm{al},{ }^{19}$ with deep nuclear gray injury assigned a basal ganglia score and cortical/white matter injury assigned a watershed score. Patients were also assigned a predominant pattern of injury classification according to Miller et $\mathrm{al},{ }^{20}$ which additionally classified patients with nonwatershed focal/multifocal white matter injury (Fig 1).

\section{ASL Image Processing}

Global CBF was calculated from the cerebral perfusion maps with the use of FSL software (http://www.fmrib.ox.ac.uk/fsl). Off-line analysis of CBF was validated by repeating measurements on the CBF maps generated by using Functool software on the Advantage Windows Workstation (GE Healthcare) with comparable results. After co-registering corresponding T2 images to the CBF maps, regional CBF was measured with the use of Medical Image
Processing, Analysis and Visualization (MIPAV) software (National Institutes of Health, Bethesda, Maryland; http://mipav.cit. nih.gov) by manually placing ROIs (each measuring 95-126 $\mathrm{mm}^{3}$; average, $104 \mathrm{~mm}^{3}$ ) over the basal ganglia, thalamus, and anterior and posterior parietal white matter (Fig 2). Corresponding right- and left-sided ROI measurements were averaged for analysis. Measurements were performed by a single investigator (A.N.M.) who was blinded to the patient clinical information and to subject condition (ie, cases versus controls). Intrarater reliability was assessed by repeating ROI placement and measurements in 15 ( $n=$ 6 controls, $n=9$ cases) randomly selected subjects and determining the intraclass correlation coefficient.

\section{Statistical Analysis}

Descriptive statistics include mean \pm standard deviation values (unless otherwise noted) for continuous variables and frequencies for categoric variables. After normality of data distributions was confirmed by Shapiro-Wilk tests, univariate analyses with independent-samples $t$ test and $\chi^{2}$ test for categoric variables were performed to evaluate differences between groups. The relationship between ASL measures and injury on MR imaging was explored by means of backwards stepwise regression modeling, which was used to identify most significant variables from potential covariates including birth weight, gestational age, sex, hemat- 


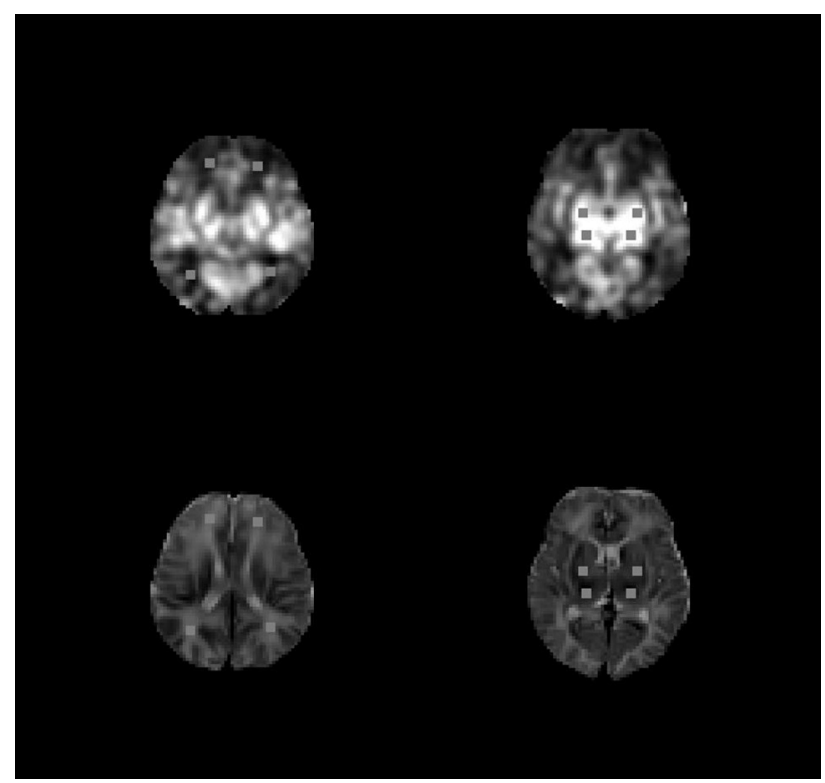

FIG 2. ROIs on axial T2 images (lower panels) co-registered to corresponding CBF maps (upper panels). ROI locations included anterior and posterior white matter at the upper level of the body of the lateral ventricles (right panels) and basal ganglia (in the putamen) and ventrolateral thalamus (left panels).

ocrit level, temperature, use of sedation during MR imaging, and age at MR imaging. The final models included variables with apparent significance across models. Adjustments for multiple comparisons were not made in these exploratory analyses. Statistical analysis was performed with SPSS 18.0 for Windows (SPSS, Chicago, Illinois).

\section{RESULTS}

\section{Baseline Characteristics of the Cohort}

Eighteen infants with HIE and 18 control infants were included in this study. An additional 3 infants treated with hypothermia underwent ASL imaging but were excluded from the analysis because of motion artifact $(n=1)$, imaging performed before the target age $(n=1)$, and presence of non-HIE injury (ie, middle cerebral artery territory stroke) recognized on qualitative imaging $(n=1)$. As expected, infants with HIE presented with low Apgar scores of 2, 4, and 5 at 1, 5, and 10 minutes, respectively. Most presented with moderate encephalopathy, both by clinical grading and electroencephalographic background pattern. Baseline characteristics including birth weight, gestational age, sex distribution, and age at MR imaging were similar between cases and controls (Table 1 ).

\section{Comparison of CBF in Infants with HIE Versus Control Infants}

Mean global CBF was significantly higher in HIE cases compared with controls (HIE, $24 \pm 5$ versus control, $19 \pm 2 \mathrm{~mL} / 100$ g per minute, $P=.001)$. This difference was largely attributable to a marked increase in regional CBF to the basal ganglia (HIE, $52 \pm$ 19 versus control, $31 \pm 5 \mathrm{~mL} / 100$ g per minute, $P<.0001)$ and thalamus (HIE, $52 \pm 19$ versus control, $29 \pm 6 \mathrm{~mL} / 100 \mathrm{~g}$ per minute, $P<.0001$ ), whereas anterior white matter differed to a smaller degree between groups (HIE, $12 \pm 3$ versus control, $10 \pm$
Demographic and clinical characteristics of the study group

\begin{tabular}{|c|c|c|c|}
\hline & $\begin{array}{l}\text { Hypothermia } \\
\qquad(n=18)\end{array}$ & $\begin{array}{l}\text { Healthy } \\
\text { Control } \\
(n=18)\end{array}$ & $P$ Value \\
\hline Birth weight, $\mathrm{kg}^{\mathrm{a}}$ & $3.26 \pm 0.68$ & $3.52 \pm 0.36$ & .176 \\
\hline Gestational age, weeks ${ }^{a}$ & $38.8 \pm 1.5$ & $39.5 \pm 1.5$ & .163 \\
\hline Sex, $n$ (\% male) & $9(50)$ & $8(44)$ & .738 \\
\hline \multicolumn{4}{|l|}{ Apgar scores } \\
\hline 1 minute & $2(0-6)$ & $8(3-9)$ & $<.001$ \\
\hline 5 minutes & $4(1-9)$ & $9(8-9)$ & $<.001$ \\
\hline 10 minutes $^{\mathrm{b}}$ & $5(2-8)$ & $\mathrm{n} / \mathrm{a}$ & \\
\hline Initial $\mathrm{pH}^{\mathrm{c}}$ & $7.03(6.74-7.35)$ & $n / a$ & \\
\hline \multicolumn{4}{|c|}{ Encephalopathy grade, $n(\%)$} \\
\hline Moderate & $15(83)$ & & \\
\hline Severe & $3(17)$ & & \\
\hline \multicolumn{4}{|l|}{ EEG at $18-24$ hours $^{d}$} \\
\hline Burst suppression & $2(12)$ & & \\
\hline Discontinuous & $13(76)$ & & \\
\hline Continuous & $2(12)$ & & \\
\hline \multicolumn{4}{|l|}{ EEG after rewarming } \\
\hline Discontinuous & $8(50)$ & & \\
\hline Continuous & $8(50)$ & & \\
\hline Electrographic seizure & $4(22)$ & & \\
\hline MRI day of life & $9(7-13)$ & $11(7-22)$ & .086 \\
\hline
\end{tabular}

Data are presented as median (range) except where noted.

${ }^{\text {a }}$ Mean $\pm S D$.

${ }^{b}$ Documented for 14 of 18 patients.

'Documented for 17 of 18 patients.

${ }^{\mathrm{D}}$ Data available for 17 of 18 patients.

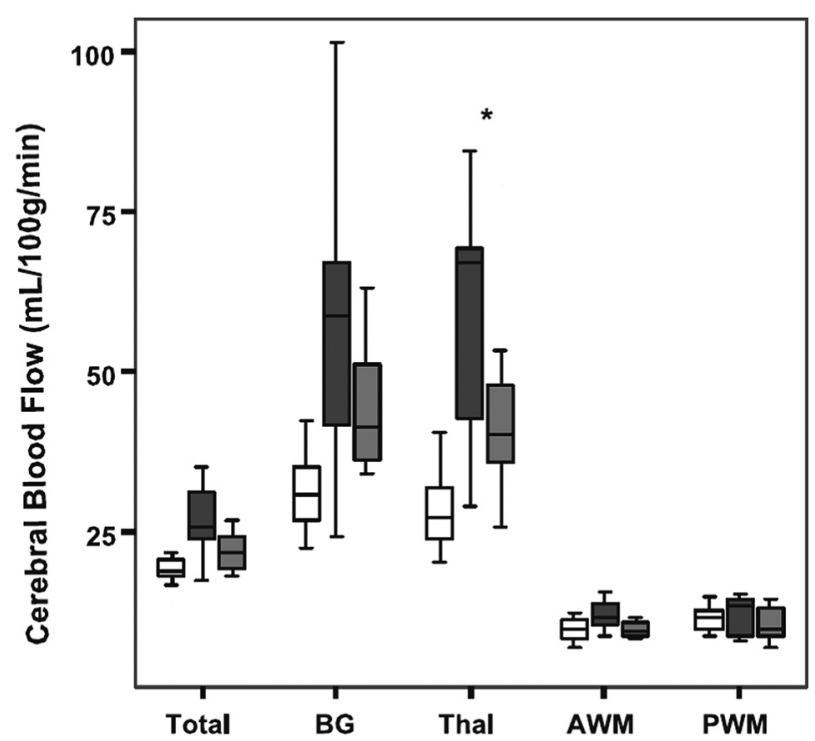

FIG 3. CBF differences by group for total brain, basal ganglia (BG), thalamus (Thal), anterior white matter (AWM), and posterior white matter (PWM). Box-and-whisker plots show median, interquartile range, and values within \pm 1.5 of interquartile range. White boxes represent control infants, dark gray boxes represent infants with HIE with normal MR imaging, and light gray boxes represent infants with $\mathrm{HIE}$ with injury on MR imaging. Overall, the HIE cohort had higher total $\mathrm{CBF}$ and regional $\mathrm{CBF}$ in the $\mathrm{BG}$, Thal, and $\mathrm{AWM}$ compared with controls $(P<.05)$. When stratified by qualitative injury on MR imaging, infants with HIE with injury did not significantly differ from controls $(P>.05)$. Differences between infants with HIE with and those without injury on MR imaging were significant in the Thal after controlling for covariates $\left({ }^{*} P=.041\right)$.

$2 \mathrm{~mL} / 100$ g per minute, $P=.045)$. Posterior white matter CBF did not differ significantly between groups (Fig 3 ). Regional measurements were highly reproducible, as demonstrated by significant 


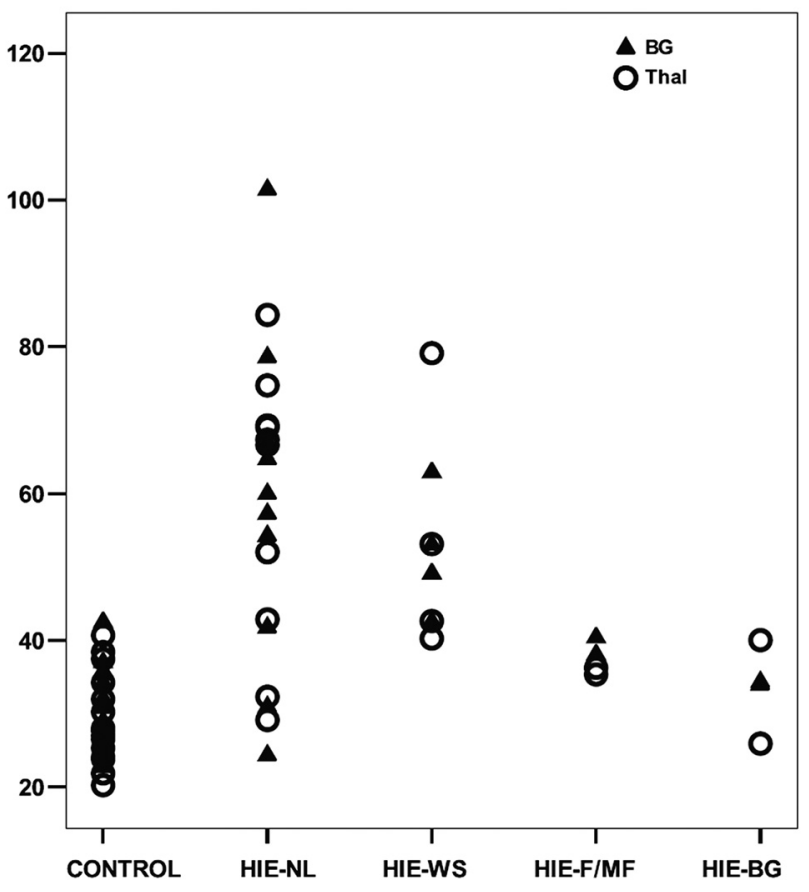

FIG 4. Regional CBF in the basal ganglia (triangles) and thalamus (open circles) by pattern of injury on MR imaging. Patients with HIE with normal (NL) MR imaging or watershed (WS) pattern of injury had relative hyperperfusion compared with controls. This was absent in infants with HIE with focal/multifocal white matter injury (F/MF) and basal ganglia predominant injury (BG).

intraclass correlation coefficient ranging from 0.807 (95\% confidence interval, $0.406-0.938, P=.003)$ for anterior white matter measurements to 0.999 (95\% confidence interval, $0.998-1.0, P<$ $.0001)$ in the basal ganglia.

\section{Relationship Between Brain Injury on Conventional MR Imaging and CBF Measures}

Qualitative injury on MR imaging was observed in 8 of 18 (44\%) infants with HIE. Four infants had watershed injury, whereas the remainder had focal/multifocal white matter injury $(n=2)$ or basal ganglia $(n=2)$ injury. CBF was lower in the infants with HIE with injury on MR imaging compared with those infants with HIE with normal MR imaging (Fig 3). This difference was statistically significant in the thalamus (B - 13.6; SE 6; 95\% confidence interval, -26.6 to $-0.67 ; P=.041)$ after controlling for $\operatorname{sex}(P>.05)$, sedation during MR imaging $(P>.05)$, and age at MR imaging $(P>.05)$ in a multiple regression model. Lower CBF was observed in the infants with HIE with focal/multifocal and basal ganglia injury, whereas some infants with watershed and most infants with normal MR imaging had relative hyperperfusion compared with controls (Fig 4).

\section{Relationship Between NIRS and Brain Injury on Conventional MR Imaging}

Complete NIRS data were available for 13 of 18 infants, including 5 infants with MR imaging injury. Infants with MR imaging injury had lower FTOE on day-of-life 4 (after rewarming) compared with infants without injury on week-2 MR imaging (Fig 5). These differences remained significant $(P=.04)$ after controlling for gestational age $(P>.05)$, sex $(P>.05)$, presence of electrographic

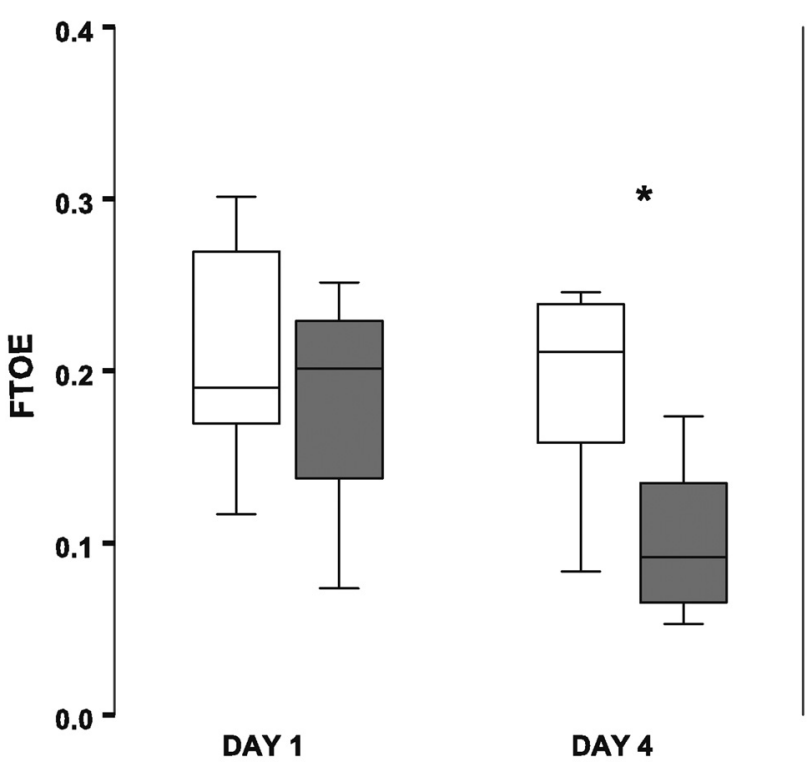

FIG 5. NIRS measurements from infants with HIE during first 24 hours of cooling (DAY 1) and after rewarming (DAY 4). Box-and-whisker plots show median, interquartile range, and values within \pm 1.5 of interquartile range. Cerebral FTOE values were significantly different after rewarming between infants with (gray boxes) and those without (white boxes) injury on MR imaging $\left({ }^{*} P<.05\right)$.

seizures $(P=.034)$, and sedation during NIRS monitoring $(P>.05)$. FTOE was not significantly correlated to ASL-CBF measures. The combined presence of low FTOE $(<0.15)$ after cooling with low CBF in the basal ganglia/thalamus $(<50 \mathrm{~mL} / 100$ g per minute) in the second week of life was significantly associated with the presence of injury on MR imaging $\left(\chi^{2}, P=.004\right)$.

\section{DISCUSSION}

In the present study, we demonstrate that disturbed CBF can be detected and quantified by ASL in hypothermia-treated infants with HIE in the second week of life. Relative hyperemia compared with controls is regional and most striking in the basal ganglia and thalamus. This hyperemia is absent in most infants with qualitative injury on conventional MR imaging, possibly suggesting pseudonormalization of $\mathrm{CBF}$ as decreased metabolic demand occurs after irreversible brain injury. ${ }^{21}$

This is the first study to evaluate ASL cerebral perfusion in infants with HIE in the second week of life after hypothermia. Postanoxic hyperperfusion has been demonstrated by ASL in pediatric and adult patients. ${ }^{22}$ Its use in neonates has been limited to a few investigations of infants with congenital heart disease ${ }^{23,24}$ and preterm infants. ${ }^{25}$ Wintermark et al ${ }^{26}$ reported 1 other small series of neonates with HIE, some of whom were treated with hypothermia. ASL was performed early in the first week of life and demonstrated marked hyperperfusion in the basal ganglia and thalamus in babies with HIE, similar to findings of the current study. However, in contrast to our findings, these investigators found CBF to be higher in infants with HIE with injury on MR imaging compared with those without injury. These differences may be attributable the timing of imaging in relation to the progression of injury. Several studies that used alternative measures of cerebral hemodynamics have associated early hyperperfusion after asphyxia with poor outcome. ${ }^{11-13,15,16}$ Few studies have in- 
vestigated the duration and evolution of this hyperperfusion response. Ilves et $\mathrm{al}^{27}$ performed serial CBF velocity measures in babies with HIE and reported that infants with severe HIE had an initial marked increase in CBF velocity followed by a decrease by 21 days compared with controls. Our findings that infants with MR imaging injury had lower CBF in the basal ganglia and thalamus by the second week of life may reflect a decreased metabolic state after progression to irreversible brain injury. This is consistent with PET studies performed at a median age of 11 days in infants with HIE, which demonstrated lower cerebral glucose metabolism in infants with severe HIE and poor outcome at 2 years. $^{21}$

It is also possible that our findings differed from Wintermark et $\mathrm{al}^{26}$ because of variable patterns of injury observed in the 2 study groups. We demonstrated that hyperperfusion is absent in babies with focal/multifocal and basal ganglia injury and variable in babies with watershed injury. This may represent variable timing of injury evolution (ie, hyperperfusion response in more mild watershed injuries takes longer to decrease or normalize after injury) or that regional perfusion response depends on mechanism (and pattern) of injury. It is of interest that the prior investigators noted 2 infants in their series with basal ganglia injury who had low $\mathrm{CBF}$ in the first few days of life. ${ }^{28}$ It is possible that some infants with basal ganglia injury may not have adequate reperfusion after the primary insult, leading to more immediate cell death, lower cerebral glucose metabolism, and persistently low regional $\mathrm{CBF}$. The true pattern and evolution of regional $\mathrm{CBF}$ response to injury cannot be elucidated by this cross-sectional study but may only be answered by serial imaging studies (now ongoing at our institution). It is clear, however, that interpretation of ASL CBF measures should be performed in the context of timing from insult and findings of injury on qualitative MR imaging.

Global CBF is increased in infants with HIE after hypothermia compared with healthy controls and probably reflects ongoing alterations in cerebral hemodynamics in response to perinatal hypoxia-ischemia. The complex cascade of secondary injurious processes known to occur during reperfusion ${ }^{6}$ makes investigation into the duration, evolution, and pattern of disturbances in $\mathrm{CBF}$ important. Continued hyperperfusion after HIE may be a harbinger of ongoing response to injury and has the potential to identify candidates for late rescue therapies. MR imaging-ASL offers a noninvasive method to interrogate cerebral blood flow while providing anatomic resolution to evaluate regional differences in cerebral perfusion. It can be added to routine brain MR imaging studies that are standard of care for diagnosis of subacute brain injury in babies with HIE. ${ }^{5,29}$ Although our study did not include another measure of CBF to corroborate our ASL measures, it is reassuring that our ASL values of global CBF in control neonates are consistent with ranges reported in the literature for normal neonates (ie, $10-20 \mathrm{~mL} / 100$ g per minute) ${ }^{30}$ In addition to providing quantitative estimation of CBF, ASL perfusion patterns can provide insight into the physiologic alterations that continue after a perinatal brain insult and the associated potential targets for late cerebral rescue and preventative interventions. We observed that regional hyperperfusion is most pronounced in the deep nuclear gray structures, known to be the most metabolically active region in the term neonate brain. ${ }^{28}$ This perfusion pattern is consistent with prior studies with the use of $\mathrm{PET}^{21}$ and perfusion MR imaging $^{31}$ in neonates during the first week of life after asphyxia. Regional hyperperfusion to the basal ganglia and thalamus may contribute to the selective vulnerability that is well described in infants with HIE after sentinel asphyxial events. ${ }^{32}$

Low CBF measures in the second week of life may represent normal neonate values versus significant brain injury and are problematic when interpreting $\mathrm{CBF}$ data in isolation. For this reason, we included available NIRS data to evaluate whether additional cerebral hemodynamic measures would facilitate differentiation of patients with injury. These values were collected retrospectively and thus were limited by incomplete available data for all infants. It is also recognized that spot measurements are less robust than more frequently sampled data and that the average hourly measurements used in our analysis is a crude summary variable when considering the high variability of individual measures $^{33}$ and the continuous nature of NIRS monitoring. Despite these limitations and consistent with prior studies, ${ }^{34,35}$ we observed a significantly lower FTOE in infants with HIE with injury on MR imaging compared with those with normal MR imaging. Combination of the early indicators of cerebral hyperperfusion with the later decrease in CBF characterizes the evolution of perfusion response leading to brain injury in these patients. It would be of additional interest to evaluate NIRS and ASL measures taken in closer temporal proximity to evaluate the correlation between these 2 methods of interrogating cerebral perfusion.

Our study has limitations. Our small sample size precluded more robust statistical analysis. Backwards regression analyses were used to select from several potential known covariables that can affect cerebral blood flow. We consider the results of these exploratory analyses to be hypothesis-generating for future studies. It is of interest that many patients with HIE with normal qualitative MR imaging had demonstrated hyperperfusion. The implications of this finding cannot be addressed by this study. It is possible that these infants had microstructural injury below the resolution of qualitative MR imaging. This may be further addressed by evaluating quantitative DTI measures, particularly in the first week of life before pseudonormalization may occur. ${ }^{36} \mathrm{We}$ previously reported that ADC measures did not differentiate infants with HIE with poor outcome when measured in the second week of life. ${ }^{37}$ Consistent with this, our qualitative grading of injury was based on T1 and T2 signal abnormality, whereas changes on diffusion imaging were rarely seen. Although analysis of quantitative DTI data is of interest, and particularly in correlation to ASL perfusion measures, it was beyond the scope of the current study. Future planned investigation will evaluate quantitative DTI measures of microstructural injury and their relation to ASL measures in serial studies performed in the first and second weeks of life. Finally, correlation of CBF findings to neurodevelopmental outcome is needed (and currently underway) to determine whether this hyperperfusion is a more sensitive indicator of microstructural injury that may be missed by qualitative review.

\section{CONCLUSIONS}

$\mathrm{CBF}$ is increased in babies with HIE compared with controls, particularly in the basal ganglia and thalamus, in the second week of 
life. Infants with qualitative injury on conventional MR imaging have lower CBF compared with those with normal MR imaging. Further study is needed to evaluate the timing and progression of regional hyperperfusion after perinatal asphyxia and to correlate findings with neurodevelopmental outcome.

Disclosures: An Massaro-RELATED: Grant: NIH, Comments: Clinical and Translational Science Institute at Children's National - KL2 Award. Money paid to institution.

\section{REFERENCES}

1. Shankaran S, Laptook AR, Ehrenkranz RA, et al. Whole-body hypothermia for neonates with hypoxic-ischemic encephalopathy. N Engl J Med 2005;353:1574-84

2. Gluckman PD, Wyatt JS, Azzopardi D, et al. Selective head cooling with mild systemic hypothermia after neonatal encephalopathy: multicentre randomised trial. Lancet 2005;365:663-70

3. Jacobs SE, Morley CJ, Inder TE, et al. Whole-body hypothermia for term and near-term newborns with hypoxic-ischemic encephalopathy: a randomized controlled trial. Arch Pediatr Adolesc Med 2011;165:692-700

4. Azzopardi DV, Strohm B, Edwards AD, et al. Moderate hypothermia to treat perinatal asphyxial encephalopathy. N Engl J Med 2009;361: 1349-58

5. Higgins RD, Raju T, Edwards AD, et al. Hypothermia and other treatment options for neonatal encephalopathy: an executive summary of the Eunice Kennedy Shriver NICHD workshop. J Pediatr 2011;159:851-58

6. Fellman V, Raivio KO. Reperfusion injury as the mechanism of brain damage after perinatal asphyxia. Pediatr Res 1997;41:599-606

7. Detre JA, Alsop DC. Perfusion magnetic resonance imaging with continuous arterial spin labeling: methods and clinical applications in the central nervous system. Eur J Radiol 1999;30:115-24

8. Calamante F, Thomas DL, Pell GS, et al. Measuring cerebral blood flow using magnetic resonance imaging techniques. J Cereb Blood Flow Metab 1999;19:701-35

9. van Bel F, Dorrepaal CA, Benders MJ, et al. Changes in cerebral hemodynamics and oxygenation in the first $\mathbf{2 4}$ hours after birth asphyxia. Pediatrics 1993;92:365-72

10. Rosenkrantz TS, Zalneraitis EL. Prediction of survival in severely asphyxiated infants. Pediatr Neurol 1991;7:446-51

11. Meek JH, Elwell CE, McCormick DC, et al. Abnormal cerebral haemodynamics in perinatally asphyxiated neonates related to outcome. Arch Dis Child Fetal Neonatal Ed 1999;81:F110-15

12. Pryds $O$, Greisen $G$, Lou H, et al. Vasoparalysis associated with brain damage in asphyxiated term infants. J Pediatr 1990;117:119-25

13. Rosenbaum JL, Almli CR, Yundt KD, et al. Higher neonatal cerebral blood flow correlates with worse childhood neurologic outcome. Neurology 1997;49:1035-41

14. Ilves $\mathrm{P}$, Lintrop M, Talvik I, et al. Changes in cerebral and visceral blood flow velocities in asphyxiated term neonates with hypoxicischemic encephalopathy. J Ultrasound Med 2009;28:1471-80

15. Ilves $\mathrm{P}$, Lintrop $\mathrm{M}$, Metsvaht $\mathrm{T}$, et al. Cerebral blood-flow velocities in predicting outcome of asphyxiated newborn infants. Acta Paediatr 2004;93:523-28

16. Frewen TC, Kissoon N, Kronick J, et al. Cerebral blood flow, crossbrain oxygen extraction, and fontanelle pressure after hypoxicischemic injury in newborn infants. J Pediatr 1991;118:265-71

17. Sarnat HB, Sarnat MS. Neonatal encephalopathy following fetal distress: a clinical and electroencephalographic study. Arch Neurol 1976;33:696-705
18. Holmes GL, Lombroso CT. Prognostic value of background patterns in the neonatal EEG. J Clin Neurophysiol 1993;10:323-52

19. Barkovich AJ, Hajnal BL, Vigneron D, et al. Prediction of neuromotor outcome in perinatal asphyxia: evaluation of MR scoring systems. AJNR Am J Neuroradiol 1998;19:143-49

20. Miller SP, Ramaswamy V, Michelson D, et al. Patterns of brain injury in term neonatal encephalopathy. J Pediatr 2005;146:453-60

21. Thorngren-Jerneck K, Ohlsson T, Sandell A, et al. Cerebral glucose metabolism measured by positron emission tomography in term newborn infants with hypoxic ischemic encephalopathy. Pediatr Res 2001;49:495-501

22. Pollock JM, Whitlow CT, Deibler AR, et al. Anoxic injury-associated cerebral hyperperfusion identified with arterial spin-labeled MR imaging. AJNR Am J Neuroradiol 2008;29:1302-07

23. Wang J, Licht DJ, Silvestre DW, et al. Why perfusion in neonates with congenital heart defects is negative: technical issues related to pulsed arterial spin labeling. Magn Reson Imaging 2006;24:249-54

24. Licht DJ, Wang J, Silvestre DW, et al. Preoperative cerebral blood flow is diminished in neonates with severe congenital heart defects. J Thorac Cardiovasc Surg 2004;128:841-49

25. Miranda MJ, Olofsson K, Sidaros K. Noninvasive measurements of regional cerebral perfusion in preterm and term neonates by magnetic resonance arterial spin labeling. Pediatr Res 2006;60:359-63

26. Wintermark P, Hansen A, Gregas MC, et al. Brain perfusion in asphyxiated newborns treated with therapeutic hypothermia. AJNR Am J Neuroradiol 2011;32:2023-29

27. Ilves P, Lintrop M, Talvik I, et al. Low cerebral blood flow velocity and head circumference in infants with severe hypoxic ischemic encephalopathy and poor outcome. Acta Paediatr 2009;98:459-65

28. Chugani HT, Phelps ME. Maturational changes in cerebral function in infants determined by 18 FDG positron emission tomography. Science 1986;231:840-43

29. Ment LR, Bada HS, Barnes P, et al. Practice parameter: neuroimaging of the neonate: report of the Quality Standards Subcommittee of the American Academy of Neurology and the Practice Committee of the Child Neurology Society. Neurology 2002;58:1726-38

30. Pryds O, Edwards AD. Cerebral blood flow in the newborn infant. Arch Dis Child Fetal Neonatal Ed 1996;74:F63-69

31. Wintermark P, Moessinger AC, Gudinchet F, et al. Perfusionweighted magnetic resonance imaging patterns of hypoxic-ischemic encephalopathy in term neonates. J Magn Reson Imaging 2008; 28:1019-25

32. Okereafor A, Allsop J, Counsell SJ, et al. Patterns of brain injury in neonates exposed to perinatal sentinel events. Pediatrics 2008;121: 906-14

33. Wijbenga RG, Lemmers PM, van Bel F. Cerebral oxygenation during the first days of life in preterm and term neonates: differences between different brain regions. Pediatr Res 2011;70:389-94

34. Toet MC, Lemmers PM, van Schelven LJ, et al. Cerebral oxygenation and electrical activity after birth asphyxia: their relation to outcome. Pediatrics 2006;117:333-39

35. Ancora G, Maranella E, Grandi S, et al. Early predictors of short term neurodevelopmental outcome in asphyxiated cooled infants: a combined brain amplitude integrated electroencephalography and near infrared spectroscopy study. Brain Dev 2013;35:26-31

36. Barkovich AJ, Miller SP, Bartha A, et al. MR imaging, MR spectroscopy, and diffusion tensor imaging of sequential studies in neonates with encephalopathy. AJNR Am J Neuroradiol 2006;27:533-47

37. Massaro AN, Kadom N, Chang T, et al. Quantitative analysis of magnetic resonance images and neurological outcome in encephalopathic neonates treated with whole-body hypothermia. J Perinatol 2010;30:596-603 